\title{
Underwater Vegetation Detection in High Frequency Sonar Images: A Preliminary Approach
}

\author{
Roberto BOZZANO, and Antonio SICCARDI \\ Consiglio Nazionale delle Ricerche, Istituto per l'Automazione Navale, CNR-IAN \\ Via De Marini 6, 16149 Genova, ITALY - E-mail: boz@ian.ge.cnr.it
}

\begin{abstract}
This paper describes tests carried out on sector-scan sonar images acquired by a high frequency acoustic device. Trials were performed on low reflective targets such as marine vegetation living on the sea bottom in shallow sea waters. The imaging process is performed by placing side by side the scanlines, with the assumption of a sufficient correlation between two successive scanlines, even if the acoustic transducer rotates and the vehicle carrying the head moves forward along its path. The selected approach is mainly based on non-linear and morphological operators. Results are shown, demonstrating the effectiveness of the approach.
\end{abstract}

\section{Introduction}

This paper describes tests carried out to analyse noisy sector-scan sonar images obtained by means of an imaging sonar. Sector-scanning sonar carried by underwater carriers (both autonomous and remotely operated vehicles) is considered as one of the most efficient devices for object detection, identification and recognition in marine environment, where optical visibility is often limited and underwater cameras cannot be employed even at low ranges [1][2]. They generally provide noisy 2D range-vs.-bearing images of the insonified 3D scenes. The signals received by the sonar transducer are related to the insonified targets presented to the sonar head. Moreover, troublesome effects, such as noise, reverberation and multipath, are generally present in sonar images, making them difficult to interpret [3].

This work is part of a project aiming at developing two small and low-cost instrumental packages to be carried by a small autonomous underwater vehicle [4]: one payload is devoted to measure standard oceanographic parameters, the other is designed to survey the seabed, in order to detect, identify and characterise low-strength targets as represented by benthic flora.

Side-scan sonar images, were diffusely employed to detect vegetation [5]: but, by using this type of images, only the detection and sometimes a macro-characterisation with some metres of resolution is possible [6]. Instead, a finer analysis is necessary to provide more precise measurements about marine vegetation.

For these reasons, a mechanically scanned, $2 \mathrm{MHz}$ narrow pencil beam monostatic sonar, vertically scanning a sector towards the sea bottom, is employed to acquire the backscattered signals and for the imaging process. The extremely high frequency of the sonar was selected in order to reach a suitable resolution capability and a high sensitivity to low strength targets (i.e., marine plant leaves) on the sea bottom. In fact, backscatter is mainly influenced by targets with dimensions comparable to half the employed wavelength or larger $(\lambda=1 \mathrm{~mm}$ at $f=1.5 \mathrm{MHz}$ and $\lambda=0.75 \mathrm{~mm}$ at $f=2.0 \mathrm{MHz}$, with $V_{s}=\lambda f=1500 \mathrm{~m} / \mathrm{s}$ ). Moreover, the backscatter response is also influenced by the roughness of the target surface and by the angle of incidence (measured from the vertical): a rough surface produces a strong and nearly uniform backscatter regardless 
the angle of incidence, while smooth surfaces are very angle-dependent, having strong returns only a low incident angles.

In this paper, the attention is devoted on the image generation process and on the focus of attention phase, aiming at defining an image area, possibly smaller than the full image, where to apply specific feature extraction algorithms [7].

\section{The Seabed Survey}

Underwater vegetation can be considered a natural and specially meaningful sea state of health gauge, and therefore it is deeply investigated in several coastal sites [8]. Several species of plants and algae, each with different biological and morphological characteristics, populate the seabed. In the present work, tests were mainly focused on the Mediterranean endemic Phanerogam Posidonia Oceanica (Fig. 1) extending along French and Ligurian coasts and in several other sea continental shelf sites.
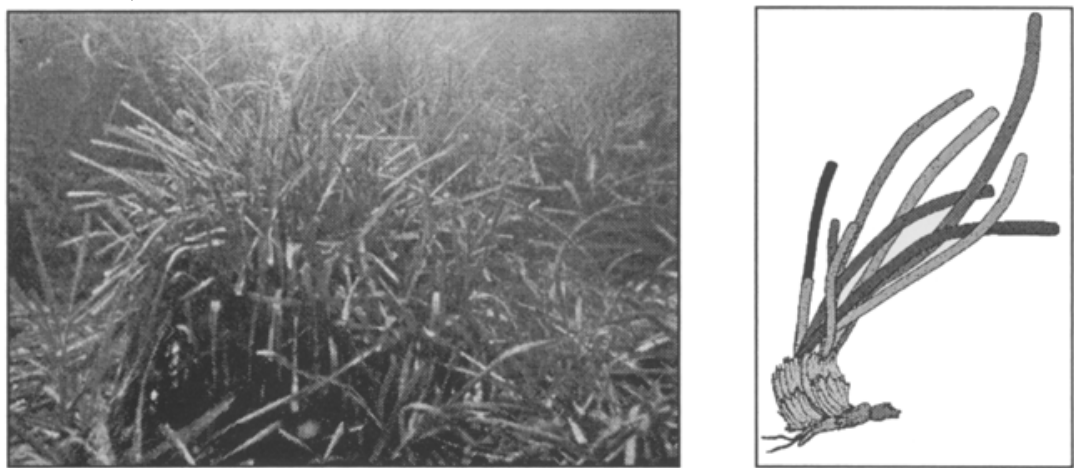

Fig. 1. (left) A dense meadows of Posidonia Oceanica (L.) Delile; (right) a drawing showing root, rhizome, and leaves of a plant.

Posidonia plays a fundamental role in the global Mediterranean coastal ecosystem since it generally forms wide underwater prairies creating a particularly suitable environment for several fish species and marine mammals [9], enriching the ecosystem with oxygen by the photosynthesis process, and making up natural barriers against the coastal erosion. Nevertheless, Posidonia is very sensitive to both natural (temperature rise, turbid water, competitive growth of the tropical green alga Caulerpa Taxifolia [10], etc.) and man-made changes (chemical and biological pollution, dikes, excavation, discharge of sewage and industrial waste, trawling): these factors often give rise to regression or quite disappearance of Posidonia beds.

\section{Sector-Scan Sonar Image Formation}

The acoustic device used to collect data about marine vegetation is a mechanically scanning high frequency sonar: its measurements result from the combination of a sequence of individual measurements made while it sweeps its nominal sensor axis through a defined sector spanning an area of interest. It can generate scans containing 800 individual measurements at equally spaced intervals of $0.45^{\circ}$ over a full $360^{\circ}$ panorama. The sonar operates by transmitting an outgoing pulse (i.e., ping) of variable duration depending on the range of the target, by listening the backscattered signal, and finally, by reorienting the transducer for the next measurement phase. 
The received echoes can be considered as a correlated sequence of scanlines, hence, a kind of image can be generated by placing side by side, in a vertical arrangement, each acquired scanline: the seabed vegetation can be viewed into a bi-dimensional domain with the axes representing the magnitude of the backscatter (in the range $0 \div 80 \mathrm{~dB}$ ) at a given distance from the sonar (vertical axis) and at a given location along the path of the underwater vehicle (horizontal axis).

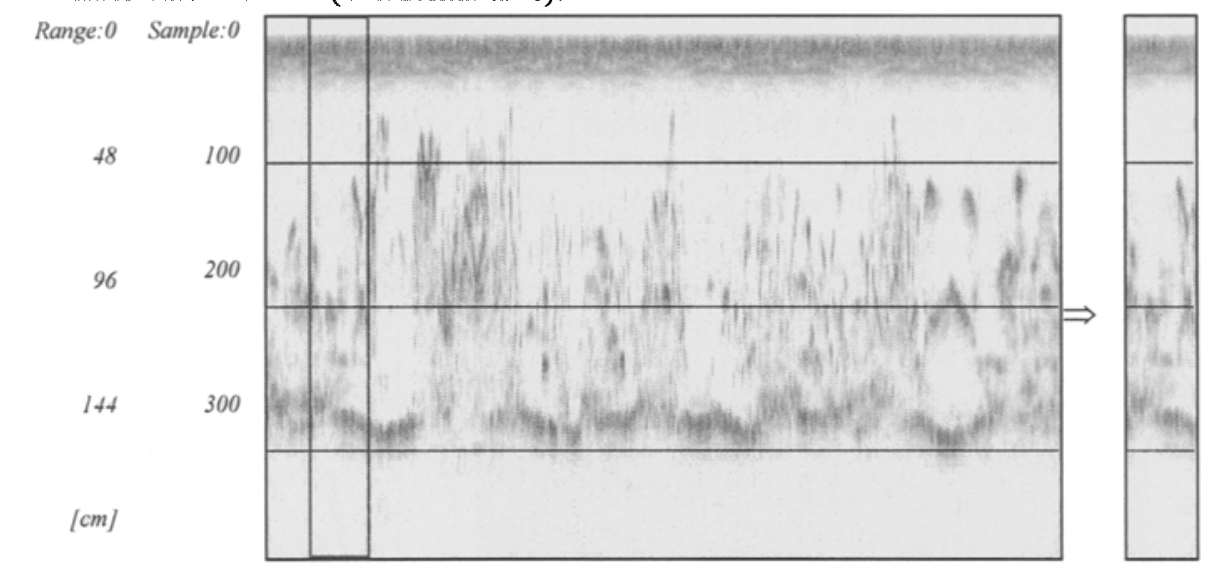

Fig. 2. An image acquired in a tank populated by Posidonia Oceanica plants on a sandy bottom.

Figure 2 shows a sonar image acquired in a tank of the "Acquario di Genova" populated by Posidonia Oceanica plants living on a few-centimetres height sandy substratum. The sonar, located at about $130 \mathrm{~cm}$ far from the sandy bottom just under the water surface, vertically scans a sector at the best angular $\left(0.45^{\circ}\right)$ and spatial ( 4.8 $\mathrm{mm}$ ) resolution. A narrow strip composed by 40 successive scanlines corresponding to the pointed out vertical rectangular zone has been extracted.

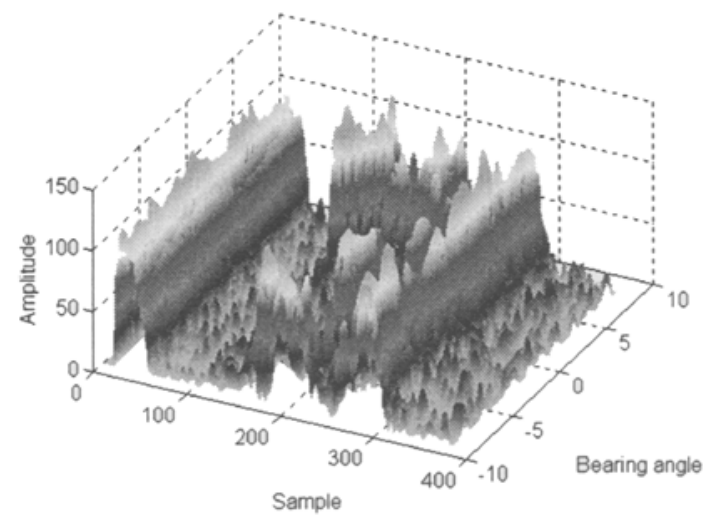

Fig. 3. Representation of the sequence of 40 successive scanlines corresponding to a narrow strip of an image as time (i.e., sample) and bearing angle vary $\left(0.45^{\circ}\right.$ resolution).

Figure 3 shows the signals referred to the above extracted strip in the time domain (i.e., one sample every $6.4 \mu \mathrm{s}$ ) as the bearing angle varies. As shown, low contrast and a preferred vertical direction are basic factors that have to be considered when the processing algorithms are selected. 
Moreover, vertical and horizontal resolutions are not a-priori fixed. For the vertical resolution, both the transmitted pulse length and the ratio between the selected range and the number of acquired samples must be considered. By sampling the backscattered echoes at the highest available rate, it assures a nominal range resolution of $4.8 \mathrm{~mm}$. However, the sonar head can handle only 1500 samples at the most: this characteristic involves a limitation about the maximum available range in order to maintain the best resolution: it is currently fixed at about 7 metres from the device. But, the highest is the number of acquired samples and the vertical resolution, the longer is the time spent to acquire the scanline data (surely depending also from the communication channels rate)and the worst is the horizontal resolution, since while the head keeps silent to store one scanline the vehicle carrying the head moves forward along its path. This involves a poor correlation between the columns of the image. So, a balance between the desired vertical resolution and the sampling interval on the seabed (i.e., the horizontal resolution) has to be reached.

\section{The Focus of Attention Procedure}

The amplitude of the received signal depends on many factors, such as the orientation of the transducer (i.e., the angle of incidence of the sonar beam with the target), the socalled target strength [11], and the transmission losses during propagation [11].

Thanks to the extremely high transmitting frequency and the narrow beam $\left(0.9^{\circ}\right)$ of the sonar, the dependence from the angle of incidence is not really important. It is however true, that the returns from a fixed small area on the sea bottom are very different if looked directly on the perpendicular direction or with a different grazing angle. Even the transmission loss is really not a fundamental parameter in the presented application since the distance between the acoustic source and the target is very short. The most important factor strongly influencing the acquired data is the target strength: even if it cannot be accurately measured, it is evident from the experimental observations that Posidonia Oceanica leaves ( $1 \mathrm{~cm}$ wide in the average), doesn't offer a high target strength value for themselves except when gas bubble are contained in the meadows. As a consequence, images have shown a narrow dynamic range (150 grey levels $-50 \mathrm{~dB}$ at the most) with a lot of spurious echoes, noise and multiple reflections.
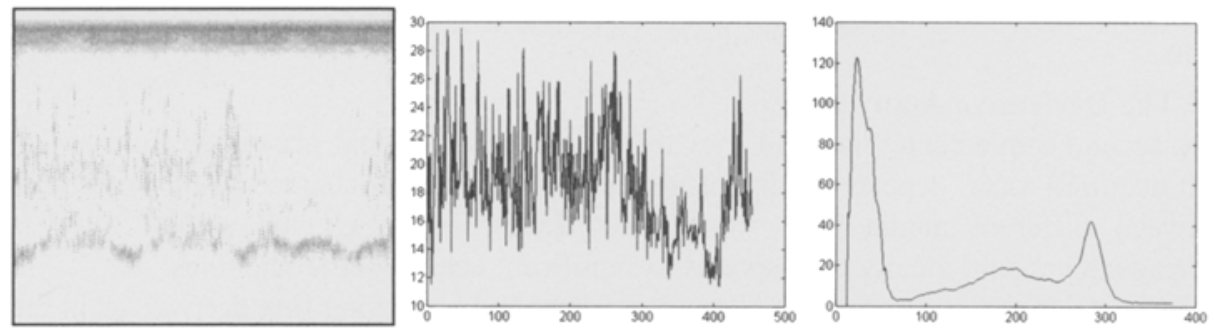

Fig. 4. (left) A sonar image and (centre) its vertical and (right) horizontal projections.

The definition of the image portion containing significant echoes, either referred to the bottom or to the eventually present vegetation, must consider these restrictions.

In order to detect the area of interest within sonar images, a simple technique (as described in [3]), partially based on the image histogram, was proposed. It can be applied only when good quality images (dynamic range and resolution) are available, but poor performances are obtained with the considered set of images. 
Figure 4 shows a test image and its vertical and horizontal projections obtained by averaging the pixel amplitude along the columns and the rows of the image, respectively: it can be seen that the vertical projection appears rather flat being the maximum range of about 18 levels. In the horizontal projection, the first peak corresponds to the transmitted pulse, while the second one detects the sea bottom. Unfortunately, this is true only if the sonar scans the seabed at a constant distance, otherwise the bottom echoes are no more aligned along neighbouring rows and the peak in the horizontal projection disappear, being mixed with the remaining signal.

By considering the above mentioned considerations, we tested two simple approaches aiming at defining a region of interest within a sonar image: the first method is composed by a median filter [12] followed by morphological operations [13] and adaptive thresholding, the second directly deals with the sonar signal by computing the derivatives along the two main axes and then by fusing them.

\subsection{The Non-Linear Approach}

The first test was conducted by spatial filtering under the assumption of a sufficient correlation degree between the image columns (i.e., scanlines). It is to note that if the sonar head is positioned at a fixed location and pointed at a flat surface with the highest angular resolution, two successive circular prints (each with diameter of $4.712 \mathrm{~cm}$ at 3 meters height) are overlapped for about half their area. Nevertheless, in real operative conditions, the head cannot be considered fixed, and the assumption about the correlation between the scans can be still considered as valid only by suitably calibrating the following set of parameters: speed of the vehicle carrying the sonar, number of samples vs. target distance, width and resolution of the angular sector to be scanned, and transmission rate.

The median filter is a rank filter which select as output the middle-ranking value from a defined neighbourhood. It is particularly efficient to remove impulsive noise in the images. The non-linear morphological filtering deals with the geometric structure within the image by means of a structuring element (SE). The aim is to quantify how that SE fits (or does not fit) within the image: hence, it is possible to derive structural information concerning a portion of the image where the SE has been applied. Morphological filters were applied after the detection by an adaptive threshold (ranging from 5 up to $12 \mathrm{~dB}$ on the ground of the applied acoustic gain) of the supposed seabed limit.

\subsection{The Derivative Approach}

The second implemented method tries to exploit the acoustical principles from which the acquired data depend: different reflection coefficient characterising interfaces between different media (e.g., water and leaves) in which the sound pulse is propagating should ideally corresponds to significant echoes in the scanlines.

Hence, tests were performed to compute vertical and horizontal first derivatives in the image: the expected results consist in two images, each of them carrying information about targets arranged in the two principal directions (e.g., the horizontal sea bottom, and the vertical plant leaves).

\section{Experimental Results}

In the following, some test results about the described algorithms are provided. 
Figure 5 shows a test image and the obtained result after a $3 \times 5$ median filtering. It is to note how over the plant leaves and under the bottom the filtered image appears to be more uniform than in the original image, while preserving the targets clusters.
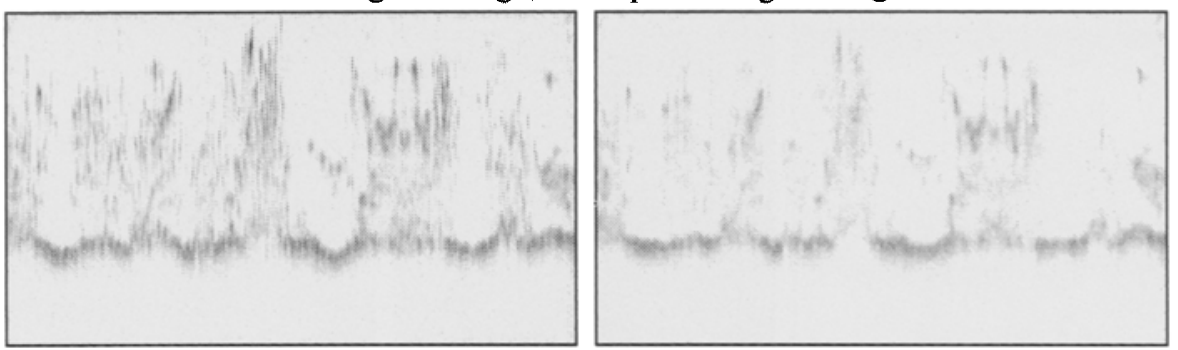

Fig. 5. (left) Original image and (right) as it appears after a $3 \times 5$ median filter ing.

The median filtered image can be thresholded to detect the sea bottom limit. Then, morphological operators (with a $7 \times 3$ disk SE) were applied. Since the low contrast and the little particulars in the images, the best operators seemed to be the dilation and the closing one, while erosion and opening seemed to loose too many information.

Figure 6 presents the morphological results obtained from the left image in fig. 4 . By considering the detected bottom limit. Then, by applying an adaptive threshold tuned with respect to the gain value used during the acoustic data collection, it is possible to detect the region of interest within a sonar image (Fig. 7).
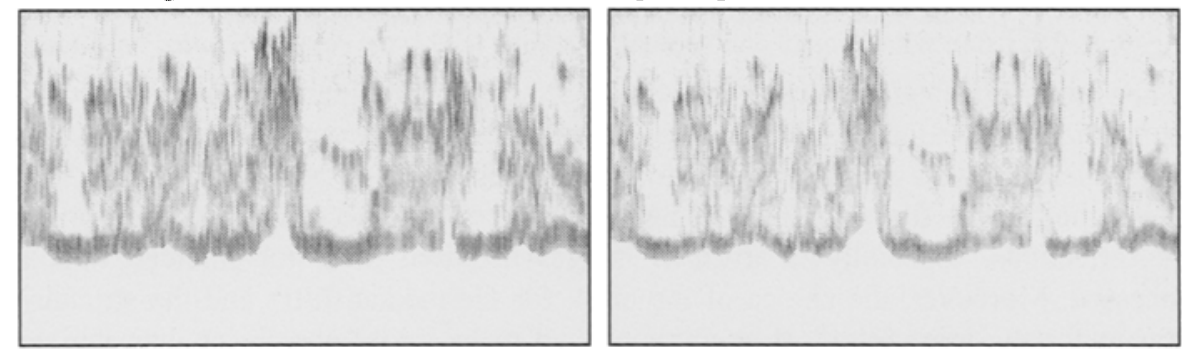

Fig. 6. Sonar image after (left) dilation and (right) closing morphological operations.
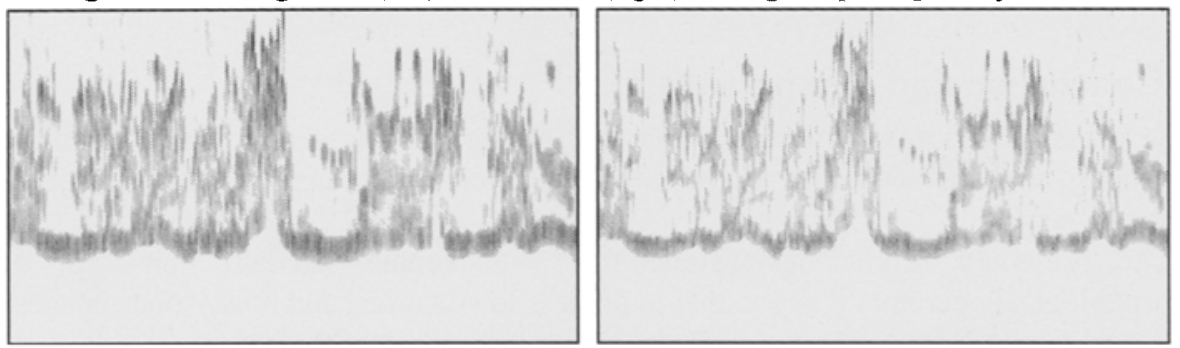

Fig. 7. Detection of plant leaves after (left) dilation and (right) closing filtering.

In the second approach we computed the horizontal and the vertical derivatives with different steps: for the horizontal edges the best results were obtained by differentiating points with 7 pixels distance along the columns (corresponding to $3.36 \mathrm{~cm}$ ), while for the vertical edges finding, a 5-pixels distance provided the more reliable results.

Figure 8 displays the original sonar image and the obtained edges along the two main axes. The basic assumption is that the main contribution to the horizontal edges derives from the echoes related to the sea bottom, and that the most significant vertical edges 
are furnished by the vertical arrangements of the plant leaves. Hence, a fusion of this information was tested, by means of a weighed sum. A new image can be generated in which each pixel value includes both the previous information (Fig. 9).
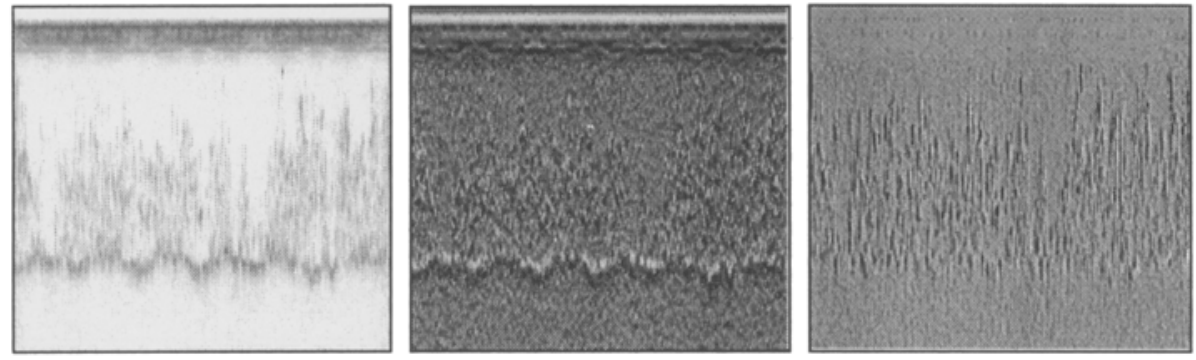

Fig. 8. (left) Original image and (centre) the extracted horizontal and (right) vertical edges.
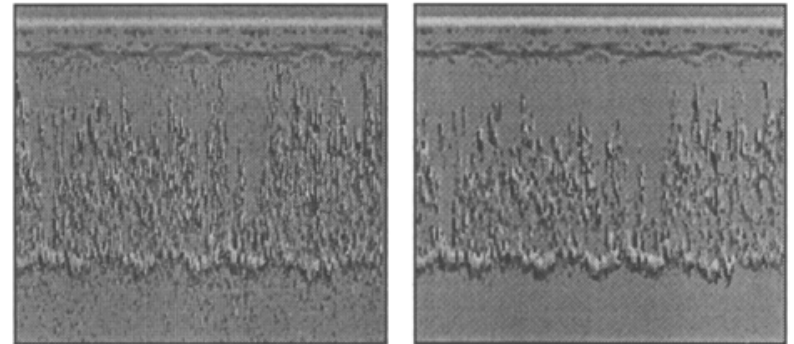

Fig. 9. (left) Fusion of horizontal and vertical edges and (right) the result after median filtering.

The results obtained by means of the two selected techniques are comparable. Both the methods provided a good separation between the meaningful portions of the backscattered signals and the subbottom or the water above the plants.

Nevertheless, the first approach seemed to be more robust to changing conditions and bad reflections, especially if artificial images acquired in a not shielded tank are processed. Moreover, the choice of the mask for the median filter and the structuring element for the morphological operator proved to be easier and faster than the steps used in the derivatives computation.

\section{Conclusion and Perspectives}

The described approach makes up the first processing phase of a system aiming at detecting and characterising the seabed vegetation by means of a high frequency imaging sonar.

The presented results demonstrate that with simple standard non-linear and morphological operators it is possible to process low-contrast and noisy sonar images in order to discriminate between significant and useless zones of the images.

The obtained results allow us to identify and characterise the seabed acquired samples: thick or sparse plants on rocky or sandy bottom areas are the main classes into which new acquired measurements can be divided into. Moreover, plants height and density can also be estimated with a certain degree of confidence from the acoustic data represented as images. Images are obtained from the data acquired in a tank of the "Acquario di Genova" with living Posidonia Oceanica plants.

Specific algorithms aiming at extracting measures were applied in order to attempt a characterisation of the seabed samples and preliminary result were described [14]. 
More refined methodologies are going to be developed and tested in order to increase the discriminative power of the characterising algorithm. Within the ambit of the MAST III project MAUVE [4], new sets of images will be acquired and processed during programmed sea trials along Ligurian and Provençal coastal sites.

\section{Acknowledgement}

This work was partially funded by the CEC MAST-III programme under the contract MAS3-CT95-0036 "MAUVE". The authors would like to thank all the partners of the project for their support and Dr. Di Natale and Dr. L. Castellano of the "Acquario di Genova" who made available the tank for the data collection.

\section{References}

[1] M.J. Chantler, D.M. Lane, D. Dai, and N. Williams, "Detection and tracking of returns in sector-scan sonar image sequences," IEE Proceedings Radar, Sonar and Navigation, Vol. 143 (3,) June 1996.

[2] J.L. Sutton, "Underwater acoustic imaging," IEEE Proceedings, Vol. 67 (4), April 1979.

[3] N. Williams, D.M. Lane, M.J. Chantler, and D. Dai, "Classification of sectorscanning sonar image sequences," Proc. of $5^{\text {th }}$ International Conference on Image Processing and its Applications IPA '95, pp. 261-265, 1995.

[4] A. Carof, "MAUVE: Miniaturised and reconfigurable instrumentation for multipurpose survey with a mini autonomous underwater vehicle," IEEE Proc. of OCEANS '96, pp. 955-960, September 1996.

[5] P. Colantoni, and P. Zucchini, "Underwater mapping of the Posidonia Oceanica prairies around the Ischia island (Naples)," Rapp. Comm. Int Mer. Medit., Vol. 25/26 (6), pp. 89-90, 1979.

[6] D.R. Carmichael, L.M. Linnet, S.J. Clarke, and B.R. Calder, "Seabed classification through multifractal analysis of sidescan sonar imagery," IEE PrOC. Radar, Sonar and Navigation, Vol. 143 (3), pp.140-148, June 1996.

[7] O. Milvang, R.B. Huseby, K. Weisteen, and A. Solberg, "Feature extraction from backscatter sonar data," Proc. of the Institute of Acoustics, Vol, 15, Part 2, pp. $157-163,1993$.

[8] J.C. Stevenson, "Comparative ecology of submersed grass beds in freshwater, estuarine, and marine environments," Limn. Ocean., Vol. 33, pp. 867-893, 1988.

[9] M.C. Buia, and L. Mazzella, "Reproductive phenology of the Mediterranean seagrasses Posidonia oceanica (1.), Cymodocea nodosa (Ucria) Aschers and Zostera noltii Hornem," Acquat. Bot., Vol. 40, pp. 343-362, 1991.

[10] A. Meinesz, J. de Vaugelas, B. Hesse, and X. Mari, "Spreading of the introduced tropical green alga Caulerpa Taxifolia in northern Mediterranean waters," Journal of Applied Phycology, Vol. 5, pp. 141-147, 1993.

[11] R.F.W. Coates, Underwater Acoustic Systems, MacMillan, 1990.

[12] R. Haralik, L. Shapiro, Computer and Robot Vision, Addison-Wesley Pub., 1992.

[13] J. Serra, Image analysis and mathematical morphology, Academic Press, 1982.

[14] R. Bozzano, and A. Siccardi, "A high frequency approach for seabed vegetation characterisation," Proc. of High Frequency Acoustics in Shallow Water conference, NATO SACLANTCEN, June 30 - July 4, 1997, in press. 